\title{
Genomic analysis of Shiga toxin-producing Escherichia coli O157:H7 from cattle and pork-production related
} environments

\author{
Peipei Zhang ${ }^{1}$, Saida Essendoubi ${ }^{2}$, Julia Keenliside ${ }^{2}$, Tim Reuter ${ }^{3,4}$, Kim Stanford $\mathbb{D}^{3,4}$, Robin King ${ }^{2}$, Patricia Lu ${ }^{2}$ and Xianqin Yang $\mathbb{D}^{1 凶}$
}

Three E. coli O157:H7 outbreaks have been attributed to contaminated pork in Alberta, Canada, recently. This study investigates the phylogenetic relatedness of $E$. coli $0157: \mathrm{H} 7$ from pigs, cattle, and pork-production environments for source attribution. Limited strain diversity was observed using five conventional subtyping methods, with most or all strains being in one subgroup. Wholegenome single nucleotide polymorphism analysis confirmed the recent ancestry of the isolates from all three sources. Most environmental isolates clustered closer with pig isolates than cattle isolates. Also, a direct link was observed between 2018outbreak environmental isolates and isolates collected from a pig farm in 2018. The majority of pig isolates harbor only one Shiga toxin gene, $s t x_{2 a}$, while $70 \%$ (35/50) of the cattle isolates have both $s t x_{1 a}$ and $s t x_{2 a}$. The results show some E. coli O157:H7 strains could establish persistence on pig farms and as such, pigs can be a significant source of the organism.

npj Science of Food (2021)5:15; https://doi.org/10.1038/s41538-021-00097-0

\section{INTRODUCTION}

Shiga toxin-producing Escherichia coli (STEC) can cause human enteric illness ranging from uncomplicated diarrhea to bloody diarrhea, with a fraction (2-22\%) of patients developing lifethreatening hemolytic uremic syndrome $(\mathrm{HUS})^{1-6}$. The predominant STEC serotype associated with human infections and outbreaks in North America is 0157:H7/NM, which also has a higher association with severe patient outcomes, compared to other serotypes ${ }^{1,7}$.

Beef has been frequently implicated in STEC 0157 outbreaks ${ }^{1,7}$ Ruminants including cattle, are regarded as a natural reservoir of STEC O157:H7 ${ }^{8}$. On the contrary, swine is not a recognized reservoir of STEC 0157:H7, and their contribution to human STEC 0157 infections has been a matter for debate ${ }^{9}$. Unlike cattle which are asymptomatic carriers, pigs can develop edema when infected with STEC strains harboring $s x_{2 e}{ }^{10}$. Outbreaks attributed to STEC 0157:H7 contaminated pork products are scarce worldwide ${ }^{11}$; however, a higher frequency has been observed in Alberta, Canada, with three outbreaks having occurred in four years (2014, 2016 , and 2018) ${ }^{12-14}$. The outbreak in 2014 was the second-largest foodborne outbreak in Canadian history with 119 lab-confirmed cases, while the 2018 outbreak had 42 confirmed cases with one death likely due to the infection with $E$. coli $0157: \mathrm{H}^{12,13}$.

A large number of studies from various geographical regions have been conducted to investigate the prevalence of STEC 0157: $\mathrm{H} 7$ in healthy pigs ${ }^{15-33}$. Most of these studies did not find this pathogen in pigs ${ }^{16-20,22,25-27,31,33}$, except for the studies from Japan $^{32}$, Ireland ${ }^{23}$, UK $^{24}$, Sweden ${ }^{28}$, Norway ${ }^{30}$, USA $^{29}$, and Canada ${ }^{15}$ where up to $2 \%$ prevalence was reported. Among these studies, a recent survey performed at 39 provincially inspected abattoirs in Alberta found $1.4 \%$ of pigs and $1.8 \%$ of pork carcasses were positive for STEC 0157:H7, and $12.8 \%$ of abattoirs had at least one positive sample ${ }^{15}$. In addition, an outbreak-related investigation in Alberta in 2018 recovered STEC 0157:H7 from pig fecal samples on the outbreak-associated farms (Keenliside, unpublished data). It is not clear whether pigs are transient carriers of STEC 0157, or some strains of the pathogen can circulate on pig farms, with pigs being the primary reservoir.

With the decrease in cost and increase in throughput for WGS, subtyping methods for surveillance and phylogenetic analysis of pathogenic bacteria have been rapidly evolving from analyzing a limited number of sites/genes in a bacterial genome to genomewide comparisons. Analysis based on single nucleotide polymorphism (SNP) and core genome (cg)/whole genome (wg) multilocus sequence typing (MLST) are the most often used WGS subtyping methods, followed by gene content analysis ${ }^{34-37}$. Despite the much improved discriminatory power of WGS-based methods ${ }^{38,39}$, it is still of value to link findings from WGS-based analysis to conventional subtyping methods, and make use of the large body of information in the literature. Thus, in the present study, we analyzed the population structure and phylogenetic relatedness of 121 STEC 0157:H7 isolates from pigs $(n=41)$, cattle $(n=51)$, and pork-production environments $(n=30)$ in Alberta, Canada using both conventional and WGS-based subtyping methods. Also, the genomes of these isolates were characterized with a focus on virulence and antibiotic resistance genes.

\section{RESULTS \\ Subtyping of $E$. coli 0157:H7 isolates using conventional methods}

All 121 E. coli 0157:H7 isolates (Table S1) were subtyped for Manning clade $^{40}$, lineage-specific polymorhpism (LSPA) ${ }^{41}$, MLST type, and Clermont phylo-group ${ }^{42}$ via in silico analysis. Most of the isolates $(115 / 121,95 \%)$ were identified to be of Manning clade 2 although cattle isolates showed more diversity (Table 1). The clades 4-7 and 9 strains share the same SNP profile for the target genes and hence cannot be differentiated by the clade typing method. Of the 121 isolates, 115 belonged to LSPA lineage I. Again, cattle isolates showed more diversity, with Cat02-Cat04 and

${ }^{1}$ Agriculture and Agri-Food Canada, Lacombe, Alberta, Canada. ${ }^{2}$ Alberta Agriculture and Forestry, Edmonton, Alberta, Canada. ${ }^{3}$ Alberta Agriculture and Forestry, Lethbridge, Alberta, Canada. ${ }^{4}$ University of Lethbridge, Lethbridge, Alberta, Canada. ${ }^{\circledR}$ email: xianqin.yang@canada.ca 
Table 1. The conventional subtyping of $121 \mathrm{E}$. coli $\mathrm{O} 157: \mathrm{H} 7$ isolates from various sources.

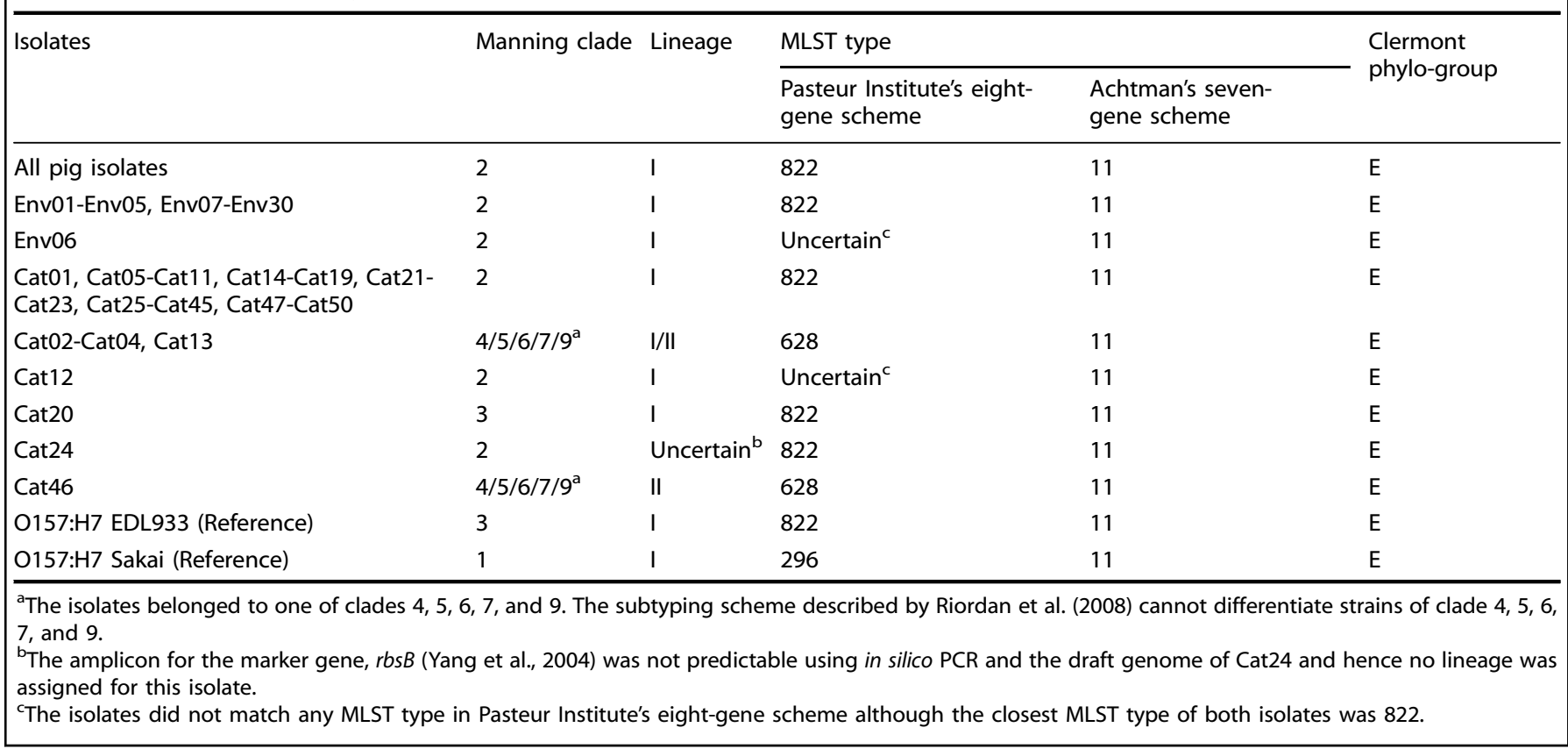

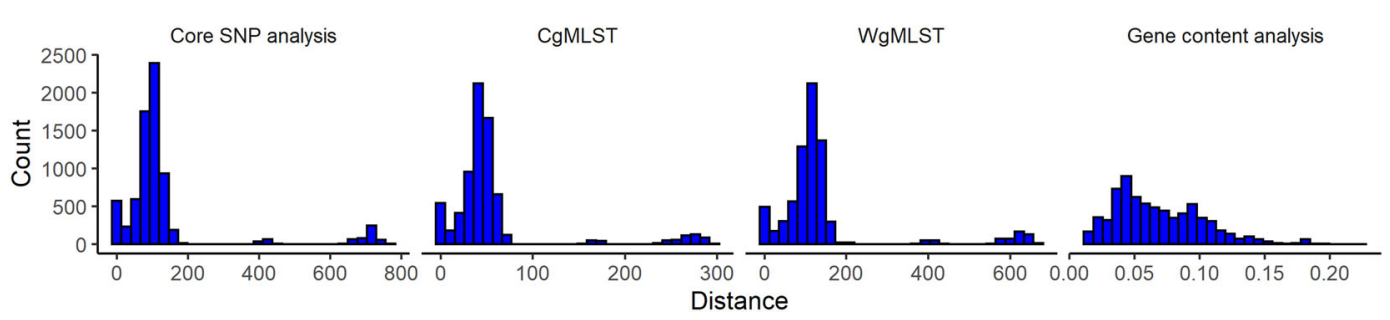

Fig. 1 Distribution of genetic distance for E. coli 0157:H7 $(n=121)$ isolated from pigs, cattle, and pork production environment. The distance was calculated from core SNP analysis (SNPs), cgMLST (allelic distance), wgMLST (allelic distance), and gene content analysis (Jaccard distance). Y-axis represents the number of combinations. There were a total number of 7260 combinations for the 121 genomes.

Cat13 belonging to lineage I/II, and Cat46 belonging to lineage II. Cat24 lacked the $r b s B$ gene and as such, could not be subtyped by LSPA $^{41}$. Using the Pasteur Institute's eight-gene scheme, Env06 (from a pig carcass) and Cat 12 did not match any MLST type in the database as the two isolates had a unique allele for the target genes polB and $i c d A$, respectively. Cat02-Cat04, Cat13 and Cat46 were identified as MLST type 628 and all the other isolates as 822 . Neither Achtman's seven-gene MLST scheme nor the Clermont's $E$. coli phylo-typing method ${ }^{42}$ was able to distinguish the isolates, with all being identified as MLST type 11 or phylo-group E, respectively (Table 1$)$.

\section{Comparison of WGS-based subtyping methods}

The phylogenetic relationships among the isolates were further mined using core SNP analysis, cgMLST, wgMLST, and gene content analysis. The core SNPs between the reference strain $E$. coli $0157: \mathrm{H7}$ EDL $933^{43}$ and the 121 isolates included 2565 sites in total. CgMLST and wgMLST included 2513 and 24952 genes, respectively. The distances among isolates were 0-770 SNPs, 0-297 alleles, 0-669 alleles, and 0.012-0.222 (Jaccard distance) for core SNP analysis, cgMLST, wgMLST, and gene content analysis, respectively (Fig. 1; Tables S2-S5).

Distance-based dendrograms constructed using the unweighted pair group method with arithmetic mean (UPGMA) showed significant correlation/similarity $(P<0.01)$ among the four dendrograms (Fig. 2). The pair-wise Cophenetic correlation coefficient (CPCC) ${ }^{44}$ among dendrograms from core SNP analysis, cgMLST, and wgMLST was between 0.995 and 0.996 (Fig. 2). The CPCC between any of the three dendrograms and that from gene content analysis was between 0.602 and 0.617 , suggesting the gene content dendrogram was only moderately correlated with the former three dendrograms. Consistent with the statistical analyses, visual comparisons showed that the phylogenetic trees from core SNP analysis, cgMLST and wgMLST clustered the isolates in a similar structure/topology while the gene content tree differed from the rest (Fig. $3 \& 4$ and S1-S2). For example, the core SNP analysis, cgMLST and wgMLST trees clustered pig and pig production environment isolates mainly into seven groups (Fig. 3, S1 and S2). In the gene content tree (Fig. 4), groups 2, 3, 5, 6, and 7 pig and pig environment isolates were in their respective distinct clusters, while groups 1 and 4 isolates were each separated into two subgroups, suggesting differences in gene gain and/or loss among the isolates within each of the two groups.

\section{Phylogenetic relatedness among the $E$. coli 0157 isolates}

The core SNP analysis was selected for interpretation of the phylogenetic relationship among the bacterial isolates. As mentioned above, environmental and/or pig isolates mostly clustered together, forming seven phylogenetic groups, 1-7, except for a few isolates (Env18, an isolate from pig feces on farm 11; Env06, from pig carcasses in plant A; Pig03, from pigs from farm 2) which clustered together with one or more cattle isolate(s) 


\section{a SNP analysis}

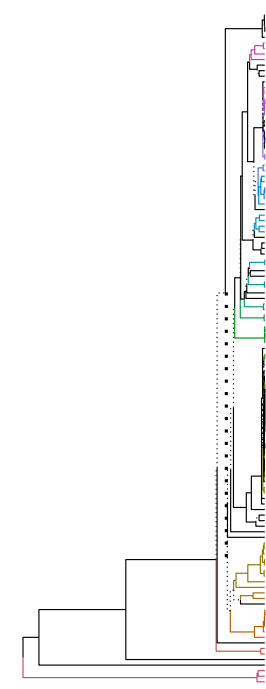

C SNP analysis
cgMLST

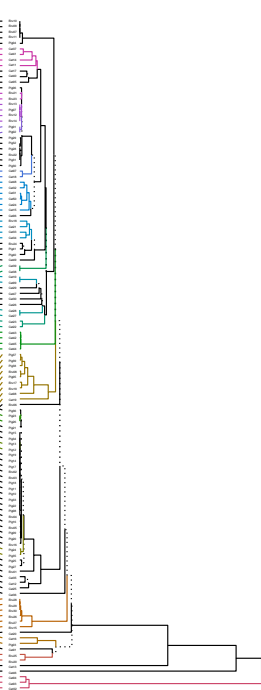

Gene content analysis

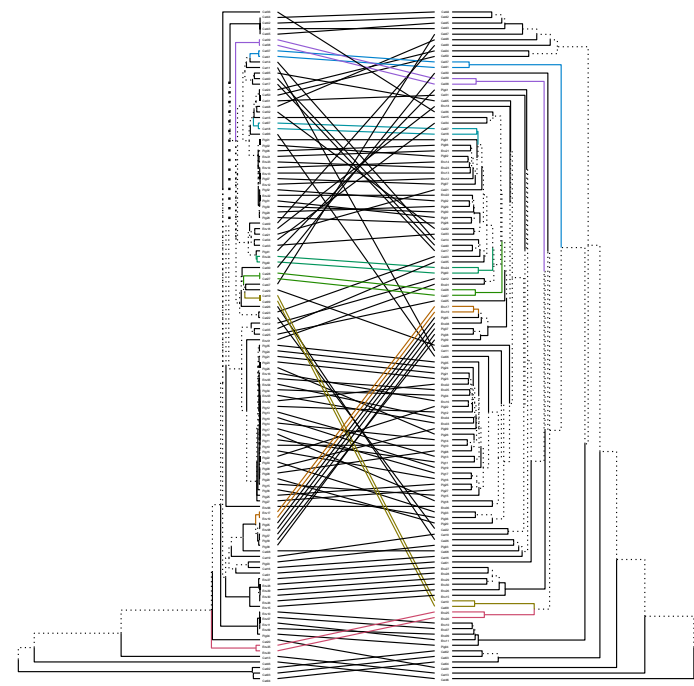

b SNP analysis

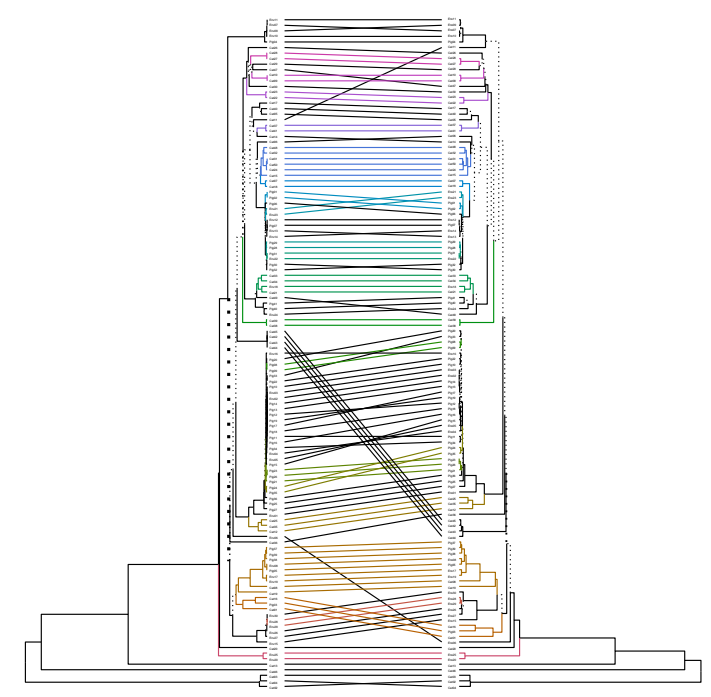

d

Cophenetic correlation coefficient

0.000 .250 .500 .751 .00

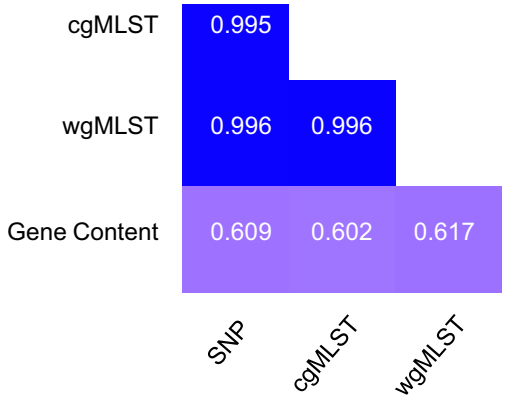

Fig. 2 Comparison of UPGMA dendrograms from four WGS-based subtyping methods. a-c shows the comparison between dendrograms produced from core SNP analysis and $\operatorname{cgMLST}(\mathbf{a}), \mathrm{wgMLST}(\mathbf{b})$, and gene content analysis (c), respectively. $\mathbf{d}$ shows the pairwise Cophenetic correlation coefficients of dendrograms. In a-c, the same tips/isolates of two trees are connected using straight lines; the connecting lines and branches are colored to highlight the sub-trees that are present in both dendrograms; the nodes which contain a combination of labels/items that are only present in one of the two trees are highlighted using dashed lines.

individually (Fig. 3; Table S2). Cut-off values of $\leq 20,>20$ and $\leq 100$ and $>100$ SNPs have been recommended to cluster bacterial isolates in food surveillance studies as closely related, possibly related and not related, respectively ${ }^{45}$. Based on this definition, most of the isolates within each of groups 1-7 were closely related (Table S2), except for Env01 (2016-outbreak isolate) which had genetic distances of 38-46 SNPs in comparison to all other isolates in group 4. Group 1-5 environmental isolates clustered closer with pig isolates than cattle isolates (Fig. $5 \mathrm{a}-\mathrm{e}$ ). The distance between environmental isolates of group 1-5 and their respective closest pig isolate(s) was 0-7, 7-8, 9-11, 0-46 and 1-17 SNPs, respectively. In contrast, the distance between group 1 and 5 environmental isolates and their respective closest cattle isolate(s) was $\geq 25,55,23,45$, and 45 SNPs. Groups 6-7 environmental isolates had distances of $\geq 84$ SNPs with either pig or cattle isolates included in this study (Fig. 5f, g). All porcine and environmental isolates had genetic distance of $\leq 100$ SNPs with at least one of the cattle isolates (Fig. S3), suggesting a recent divergence in ancestry of these isolates.

The pig and environmental isolates were recovered from pig fecal samples or environmental samples collected from 11 farms (1-11) or four meat processing plants (a-d) (Figs. 3 and 6). Closely related pig isolates were recovered from farms 1 and 4 over a period of two years and frequently from pigs on farm 9 (Figs. 3 and 6). The genetic distances between the 2018-outbreak isolates (Env02-Env05, Fig. 5d) and pig isolates from farm 9 were $\leq 10$ SNPs, indicating a strong association between this outbreak and farm 9 pigs. Both the 2014-outbreak isolates (Env15, Env26-Env30; Fig. 5f) and a 2016-outbreak isolate (Env01, Fig. 5d) had similar genetic distance to their respectively closest pig ( $\geq 93$ SNPs, 2014; $\geq 38$ SNPs, 2016) and cattle ( $\geq 91$ SNPs, 2014; $\geq 49$ SNPs, 2016) isolates. 


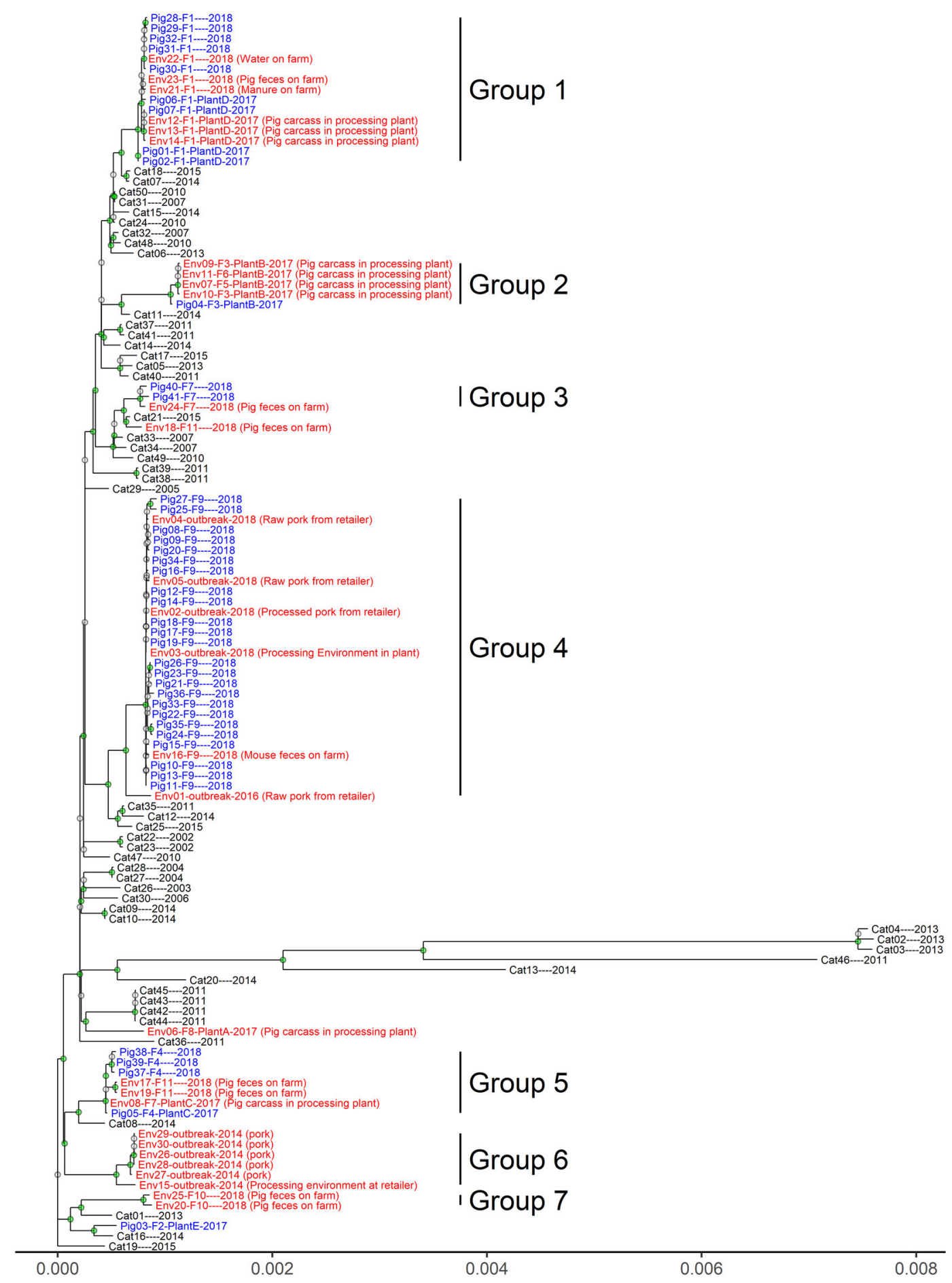

Fig. 3 A maximum likelihood tree based on core SNPs of EDL933 (Reference) and 121 E. coli 0157:H7 isolates from pigs, cattle and pork production environment. The nodes with bootstrap support value $>70$ are labeled green. The substitution(s) per site is represented in the scale bar. Each tip in the tree was labeled with the isolate's identification and, if applicable, its associated farm (F), and/or processing plant (Plant) followed by the year of collection. The pig isolates (blue) and cattle (black) isolates were recovered from fresh fecal/cecal samples of both animal species. The environment isolates (red) were recovered from various samples (information added in the parenthesis following the tree tips) collected from the pork production and distribution chain. Porcine and environmental isolates primarily clustered in seven groups in the tree, as labeled in the figure.

\section{Pan-genome}

In total, 8096 genes were found in the pan-genome of the 121 isolates, including 4052 (50.05\%) core genes (shared by all 121 isolates) and 4044 (49.95\%) accessory genes (present in between 1 and 120 isolates) (Fig. S4). The database for Clusters of
Orthologous Groups of proteins (COGs) ${ }^{46}$ was used to classify the functional categories of each gene in the pan-genome. In total, 745 core and 2871 accessory genes were not classified. Of note, among the classified genes in the accessory genome, category X (mobilome: prophages, transposons) accounted for the 


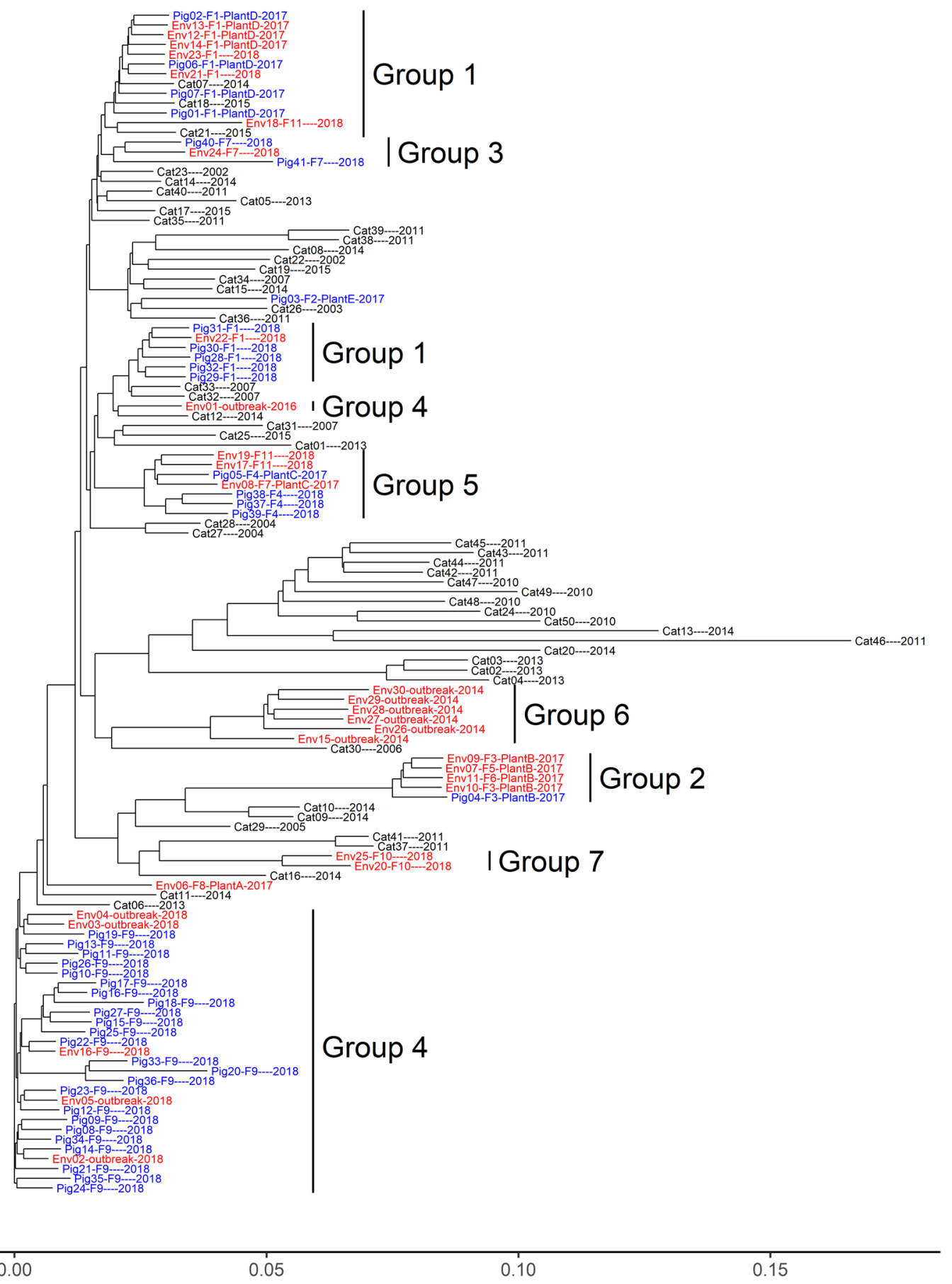

Fig. 4 A neighbor joining tree based on the gene content of $121 \mathrm{E}$. coli 0157:H7 isolates from pigs, cattle and pork production environment. The scale bar represents the Jaccard distance based on the presence or absence of genes between genomes. Each tip in the tree was labeled with the isolate's identification and, if applicable, its associated farm (F), and/or processing plant (Plant) followed by the year of collection. The isolates recovered from pig (blue) and cattle (black) fecal samples and pig production environment samples (red) were distinguished using different colors. The branches of the seven groups formed by pig and environment isolates in core SNP tree (Fig. 3 ) are labeled in text in the figure. The groups 2,3,5,6, and 7 were also clustered together in this tree, however, groups 1 and 4 were separated into two respectively subgroups.

largest proportion (38.9\%), indicating the large contribution of this category to the plasticity of $E$. coli O157:H7 genomes.

\section{Virulence genes}

Genes encoding Shiga toxin (stx) subtypes 1a, 2a and 2c were found in the 121 STEC 0157:H7 genomes, with the patterns of "1a only", "2a only", "2c only", "1a and 2a", "1a and 2c" (Fig. 6; Table 2).
Of the 41 pig isolates, $61 \%$ (25) harbored $s t x_{2 a}$ only and all the other pig isolates $(16 ; 39 \%)$ had both $s t x_{1 a}$ and $s t x_{2 a}$, while $33.3 \%$ (10) and $66.7 \%$ (20) of environmental isolates had $s t x_{2 a}$ only and stx $x_{1 a}$ and $s t x_{2 a}$, respectively (Table 2). The most abundant pattern among cattle isolates was " $1 \mathrm{a}$ and $2 \mathrm{a}$ " (35, 70\%), followed by "1a only" $(5,10 \%)$, "2a only" $(5,10 \%)$, " $1 \mathrm{a}$ and $2 \mathrm{c}$ " $(4,8 \%)$ and " $2 \mathrm{c}$ only" $(1,2 \%)$. The stx profiles correlated with their respective phylogenetic groups (Fig. 6). For example, group 1, 3, 5, and 6 
a

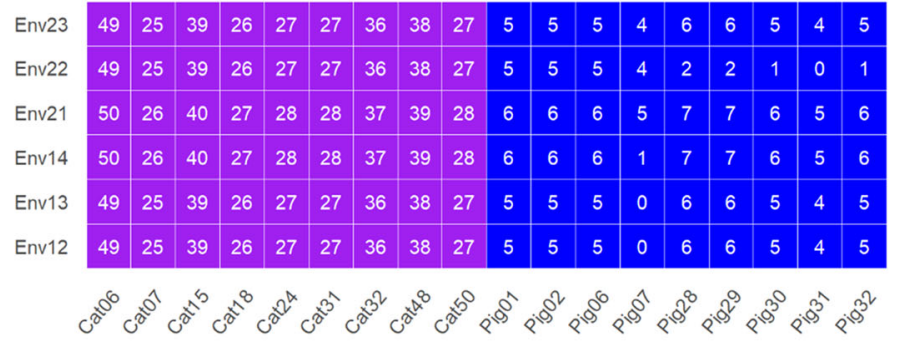

b

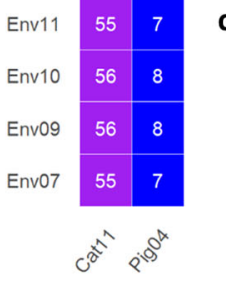

C

Env24

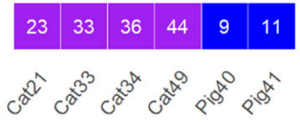

d

\begin{tabular}{llll|l|l|l|l|l|l|l|l|l|l|l|l|l|l|l|l|l|l|l|l|l|l|l|l|l|} 
Env16 & 64 & 54 & 47 & 3 & 4 & 2 & 2 & 2 & 2 & 2 & 4 & 3 & 2 & 2 & 2 & 4 & 6 & 3 & 5 & 7 & 10 & 5 & 10 & 3 & 3 & 7 & 8 \\
Env05 & 64 & 54 & 47 & 3 & 4 & 2 & 2 & 2 & 2 & 2 & 4 & 3 & 2 & 2 & 2 & 4 & 6 & 3 & 5 & 7 & 10 & 5 & 10 & 3 & 3 & 7 & 8 \\
\hline Env04 & 63 & 53 & 46 & 2 & 3 & 1 & 1 & 1 & 1 & 1 & 3 & 2 & 1 & 1 & 1 & 3 & 5 & 2 & 4 & 6 & 9 & 4 & 9 & 2 & 2 & 6 & 7 \\
Env03 & 62 & 52 & 45 & 1 & 2 & 0 & 0 & 0 & 0 & 0 & 2 & 1 & 0 & 0 & 0 & 2 & 4 & 1 & 3 & 5 & 8 & 3 & 8 & 1 & 1 & 5 & 6 \\
Env02 & 62 & 52 & 45 & 1 & 2 & 0 & 0 & 0 & 0 & 0 & 2 & 1 & 0 & 0 & 0 & 2 & 4 & 1 & 3 & 5 & 8 & 3 & 8 & 1 & 1 & 5 & 6 \\
Env01 & 66 & 56 & 49 & 39 & 40 & 38 & 38 & 38 & 38 & 38 & 40 & 39 & 38 & 38 & 38 & 40 & 42 & 39 & 41 & 43 & 46 & 41 & 46 & 39 & 39 & 43 & 44
\end{tabular}

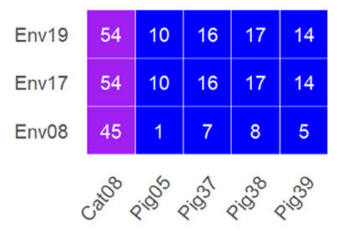

f

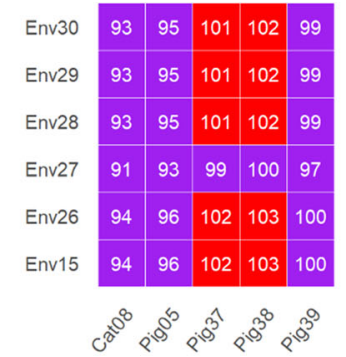

g

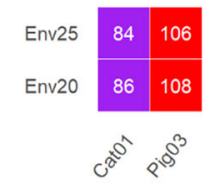

Fig. 5 The SNP distance of environmental isolates of each phylogenetic group with their closest cattle and pig isolates. a-g shows the environmental isolates of group 1-7, respectively. The SNP distances are coded with blue, purple, and red representing $<21$ SNPs, $\geq 21$ SNPs and $\leq 100$ SNP, and $>100$ SNPs, respectively.

isolates harbored both $s t x_{1 a}$ and $s t x_{2 a}$, while group 2 and group 4 (except isolate Env01 from the 2016-outbreak) had $s t x_{2 a}$ only.

Besides stx, 319 other virulence genes were found in the genomes (Table S6). The gene eae located on the locus of enterocyte effacement (LEE), encoding intimin, was found in all but Cat48 (Table S6). The gene paa encoding the porcine attaching/effacing-associated protein ${ }^{47}$ and tox $B$ encoding an adhesion protein ${ }^{48}$ were absent in only one genome, Cat13. EhaA encoding an autotransporter protein that contributes to adhesion and biofilm formation ${ }^{49}$ was found in all genomes. The genes saa and IpfA which encode an autoagglutinating adhesion or long polar fimbriae in LEE negative STEC ${ }^{50,51}$, respectively, were not found in any of the genomes. Three genes encoding potential adhesion proteins $c 3610$, cah, ih ${ }^{52}$ were absent in all phylogenetic group 2 isolates, but present in most other isolates. a-hemolysin encoding gene $e h x A^{53}$ was found in all genomes but Cat45. A heat-stable enterotoxin (EAST1) encoding gene ast $A^{54}$ was found in all isolates. Another thermostable enterotoxin gene estlA often found in enteroaggregative $E$. coli $i^{55}$ or a subtilase cytotoxin encoding gene $s u b A^{56}$ was not found in any of the genomes.

\section{Antibiotic resistance}

Of the $121 \mathrm{E}$. coli $\mathrm{O} 157 \mathrm{H} 7$ isolates, 22 (18.2\%) were predicted to be resistant to at least one of six classes of antibiotics including aminoglycoside, beta-lactam, phenicol, sulfonamide, tetracycline, and/or trimethoprim (Fig. 6). The 2014-outbreak isolates (group 6) were resistant to aminoglycosides, phenicols, and sulfonamides while the 2016- and 2018-outbreak isolates were predicted not to be resistant to any group of the antibiotics. Group 2 and 7 isolates were resistant to four and three antibiotic classes, respectively. On the contrary, group 1, 3, 4, and 5 isolates, which were associated with farm 1/plant D, farm 7, farm 9, and farm 4/farm 11/plant C, respectively, were predicted not to be resistant to any of the antibiotics examined.

\section{Plasmid sequences}

Bacterial plasmids often carry virulence and antibiotic resistance genes ${ }^{57}$. The program PlasmidFinder ${ }^{58}$ evaluates a replicon sequence as a signature to identify plasmid sequence-bearing contigs. In total, 11 variants of replicons were found in the genomes, including Col(BS512), Col156, IncFIA, IncFIB(AP001918), IncFIl, IncFII(pHN7A8), IncFII(pCoo), Incl2(Delta), Incl1-I(Gamma), IncN, and pEC4115 (Table S7).

All isolates had replicon sequences of IncFIB (AP001918) and IncFII. Isolates Cat02-04 and Cat46 all had IncFIA as well. A Blast screening against the $\mathrm{NCBI}$ nucleotide database found that the contigs containing these replicon sequences all had a $>99 \%$ identity with the megaplasmid, p0157 often present in E. coli 0157:H7 genomes ${ }^{59}$. The p0157 marker gene ehxA and relevant transport protein-encoding genes etpC-O were found in these contigs.

A larger percentage of cattle isolates $(26 \%, 13 / 50)$ carried other plasmids/replicons compared to pig isolates (2/41, 4.9\%). For pig and environmental isolates, the presence of replicons was related to their phylogenetic group with groups 2, 6, and 7 having Col(BS512) and Incl1-I(Gamma), IncFII(pCoo), IncFII(pCoo) 


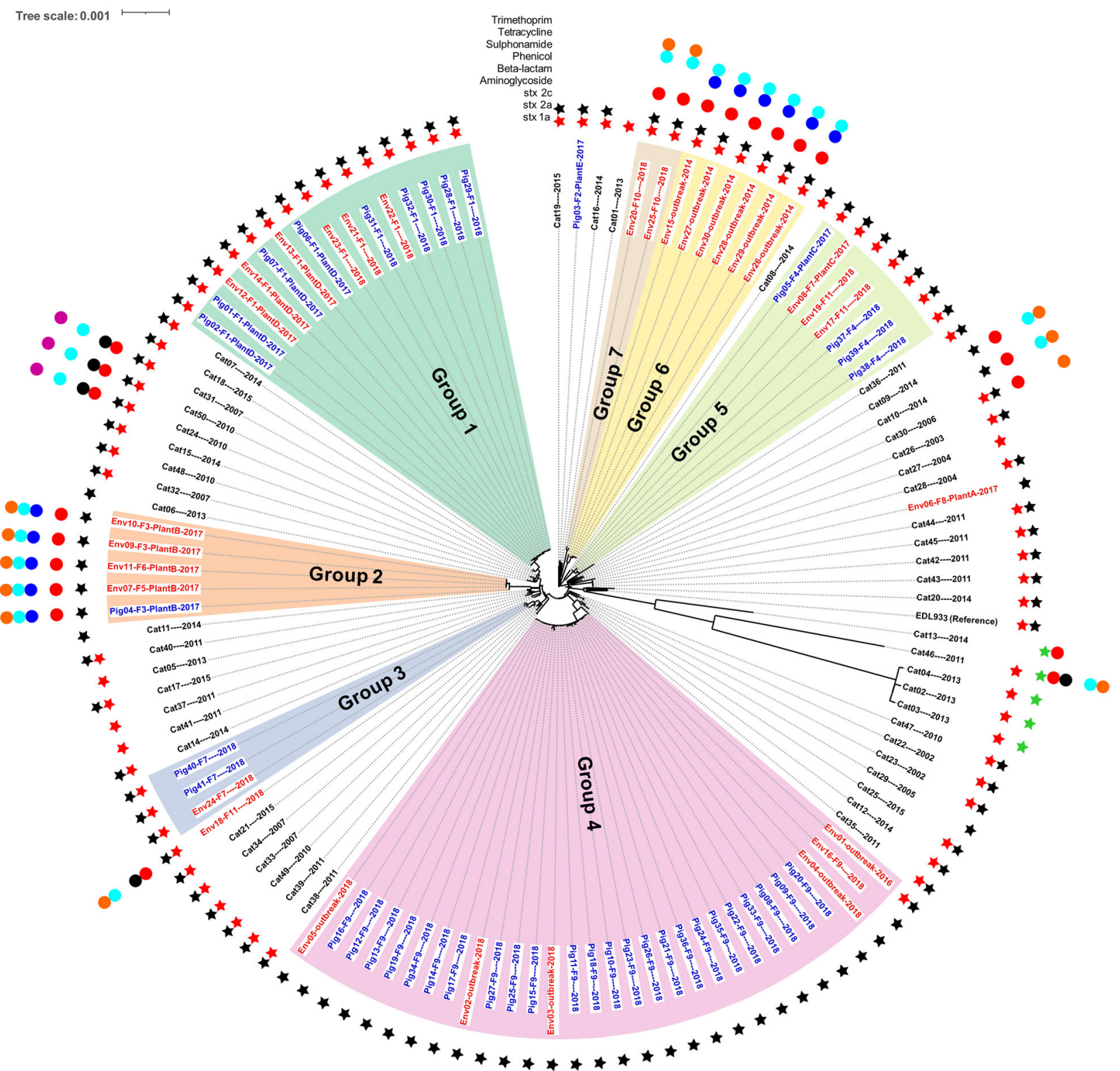

Fig. 6 The core SNP tree shown in the circular. The tip text for cattle, pig, and pig production environment isolates is print in black, blue, and red, respectively. The format is "isolate id-year of collection" for cattle isolates and "isolate id -associated farm (if available) -associated plant (if available)-year of collection" for pig and environment isolates, respectively. The seven phylogenetic groups formed by pig and environment isolates are highlighted with different colors and labeled with text. The presence of Shiga toxin-producing genes $s t x_{1 a}$, $s t x_{2 a}$, and $s t x_{2 c}$ is shown as a red, black, and green star, respectively. The resistance to classes of antibiotics for each isolate, including aminoglycoside, beta-lactam, phenicol, sulfonamide, tetracycline, and trimethoprim, is shown as a red, black, dark blue, light blue, orange, and purple circle, respectively.

Table 2. The number (percentage, \%) of E. coli O157:H7 isolates with different Shiga toxin-producing gene profile.

\begin{tabular}{lccc}
\hline & Pig isolates & Cattle isolates & $\begin{array}{l}\text { Environmental } \\
\text { isolates }\end{array}$ \\
\hline Stx $_{1 a}$ only & $0(0)$ & $5(10)$ & $0(0)$ \\
St $_{2 a}$ only & $25(61)$ & $5(10)$ & $10(33.3)$ \\
St $_{2 c}$ only & $0(0)$ & $1(2)$ & $0(0)$ \\
Stx $_{1 a}+$ Stx $_{2 a}$ & $16(39)$ & $35(70)$ & $20(66.7)$ \\
Stx $_{1 a}+$ Stx $_{2 c}$ & $0(0)$ & $4(8)$ & $0(0)$ \\
Total & $41(100)$ & $50(100)$ & $30(100)$ \\
\hline
\end{tabular}

and Incl1-I(Gamma), respectively (Table S7). The IncN replicon in Env18 (recovered from pig feces on farm 11) had resistance genes to four classes of antibiotics including $a p h\left(3^{\prime \prime}\right)-l b$ and $a p h$ (6)-Id, blaTEM-1B, sul2, and tet(A) for aminoglycosides, betalactams, sulfonamides, and tetracyclines, respectively. The plasmids with IncFII(pCoo) in group 6 isolates harbored genes encoding resistance to aminoglycosides (aadA2b and aadA1), phenicols (cm/A1) and sulfonamides (sul3). For isolates Cat50, Cat31, and Cat24, the plasmids with IncFII(pHN7A8) carried the resistance genes for aminoglycosides (aph(3")-lb and $a p h(6)-I d)$ and sulfonamides (sul2). However, in the genome of Cat30 the IncFII(pHN7A8)-containing contig only carried aminoglycoside resistance genes (aph(3")-Ib and $a p h(6)-I d)$. 


\section{DISCUSSION}

This study investigated the phylogenetic relatedness of STEC 0157:H7 $(n=121)$ recovered from pigs, cattle, and porkproduction-related environments in Alberta, Canada, using both conventional subtyping schemes and WGS-based methods. To date, a paucity of information on the genetic relationship between cattle and swine STEC strains is available in published accounts. $E$. coli 0157:H7 strains from the two animal species showed distinct randomly amplified polymorphic DNA (RAPD) patterns in a Korean study ${ }^{60}$, while similar pulsed-field gel electrophoresis (PFGE) patterns were found in a Norwegian study ${ }^{30}$. The present study observed very limited strain diversity for isolates from both animal species determined by all five conventional subtyping methods: $95 \%, 95 \%, 94.2 \%, 100 \%$, and $100 \%$ of the isolates belonging to one Manning clade (2), LPSA lineage (I), MLST type (822; Pasteur Institute's eight-gene scheme), MLST type (11, Achtman's sevengene scheme) or Clermont phylo-group (E). Genetic variation of $E$. coli 0157 can be driven by geographical locations ${ }^{61-64}$. Published data reviewed by Pianciola and Rivas ${ }^{63}$ showed that the most predominant Manning clade of $E$. coli $\mathrm{O} 157: \mathrm{H7}$ in the USA, Argentina, and Australia is 2,8 , and 7 , respectively. Strachan et al. ${ }^{64}$ showed the predominant E. coli $0157: \mathrm{H} 7$ in Canada between 1995 and 2012 is clade 2, followed by clade 3. LSPA lineage I have been reported to be predominant among cattle $E$. coli O157:H7 isolates in Canada, followed by lineage II and $1 / \mathrm{II}^{63}$, which is consistent with the findings in this study.

The four WGS-based subtyping methods all showed a much higher discriminatory power compared to conventional typing methods, as would be expected. Phylogenetic trees from the former three subtyping methods congruously separated the porcine and environmental isolates mainly into seven phylogenetic groups, with cgMLST having the lowest resolution. However, the gene content analysis further separated group 1 and group 4 into two subgroups, with different positioning for some isolates, and divided clonal isolates, especially in the phylogenetic trees based on SNP and cgMLST analysis into well-defined branches. SNP analysis uses the genome of a closely related bacterial strain as a reference and infers phylogeny among bacterial strains based on the variants in both intragenic and intergenic regions shared by both reference and query genomes ${ }^{34}$. On the other hand, cgMLST and wgMLST analyze the allelic difference of genes in the core (cgMLST) and pan (wgMLST) genomes, respectively ${ }^{34}$. For both cgMLST and wgMLST, the missing alleles are ignored in pairwise comparison and one allelic difference is counted for a given gene if two genomes have different alleles no matter how many SNPs are found in this gene. The difference in discriminatory power of SNP and cg/wgMLST was then to be expected. A gene content-based method infers the phylogeny based on the presence or absence of genes in the pan-genome of included bacterial strains and hence reflects difference in the accessory genome rather than the core genome ${ }^{36,37}$. The differences between the accessory genome tree (gene content analysis) and core genome trees (SNP and cgMLST) are in agreement with the previous observations on the plasticity of $E$. coli genomes, with horizontal gene transfer being the primary driving force for the evolution of $E$. coli ${ }^{37,65}$. The observation that some cattle isolates (e.g. Cat07 and Cat18) intermingled with pig isolates suggest that those pig isolates may have originated from cattle or a common source with cattle isolates, and that a subgroup of cattle isolates may have acquired accessory genomes to adapt to the pig gut environment.

Pightling et al. ${ }^{45}$ suggested a cutoff value of 21 and 100 SNPs for defining clonal strains and possibly related strains of $E$. coli for outbreak investigations. All porcine and environmental isolates had genetic distance of $<100$ SNPs with at least one cattle isolate included in this study, suggesting the recent common ancestry shared between E. coli 0157:H7 from both animal species. This is consistent with the low strain diversity observed by conventional subtyping methods. The genetic differences among isolates within each phylogenetic group of pig and environmental isolates are mostly less than 20 SNPs. A large number of clonal STEC 0157: H7 strains, as determined by SNP analysis, were frequently recovered from pigs (farm 9, $\leq 13$ SNPs; farm 1, $\leq 7$ SNPs). The prevalence of STEC 0157:H7 in pigs is low in most geographical areas globally and whether swine are competent carriers of this pathogen has long been debated ${ }^{9}$. A study carried out by Wöchtl and co-workers $^{66}$ reported clinical symptoms including diarrhea, dehydration, and neurological disorders in piglets infected with STEC 0157:H7. In contrast, Booher et al. ${ }^{67}$ found pigs can be persistently colonized by STEC 0157:H7 for $>2$ months. The repeated recovery of the clonal strain of STEC 0157 from pigs on individual farms over an extended period of time in the present study supports the latter of the two studies. The 2018-outbreak isolates and pig isolates from farm 9 differed by less than 10 SNPs. To the authors' best knowledge, a direct link between STEC 0157: $\mathrm{H} 7$ of pig origin and an outbreak found in this study was not reported before. The 2016-outbreak isolates and farm 9 isolates recovered in 2018 may share a recent common ancestor as they differed only by 38-46 SNPs. In addition, most (66.7\%, 20/30) environmental isolates are more closely related to pig isolates than cattle isolates. These findings suggest that pigs can be a significant source of STEC 0157 contamination on pork and in pork-production environments.

Shiga toxins, carried by prophages, are the defining feature of STEC. There are two types of Shiga toxins, Stx 1 and Stx2, each further divided into several subtypes (Stx1a and Stx1c-1e; Stx2a-2i, Stx2k, and Stx2l) based on the serological reaction and the amino acid sequence ${ }^{1}$. Stx2e can cause edema disease in pigs ${ }^{68}$. In general, Stx2 is more associated with the human disease than Stx1, with the subtype $\mathrm{Stx}_{2 \mathrm{a}}$ being often associated with patients with HUS than other subtypes ${ }^{1,69-71}$. A study of Chui et al. ${ }^{72}$ reported negative interactions between Stx1a and Stx2a, proposing that STEC 0157 isolates carrying only Stx2a are more virulent than those with both Stx1 and Stx2a. The cattle and pig/ environmental isolates had different stx gene profiles. The cattle isolates mostly carried $s t x_{1 a}$ and $s t x_{2 a}$ and this agrees with previous reports on the stx profile of STEC 0157 recovered from Alberta cattle ${ }^{73}$. On the other hand, most pig isolates carried $s x_{2 a}$ only. An Indonesian study reported that STEC 0157:H7 from pigs $(n=7)$ all had $s_{2}{ }_{2 a}{ }^{74}$; however, only $s_{2} x_{2 a}$ was examined in that study. There are a total of 14 genomes of STEC 0157:H7 pig isolates deposited in GenBank ( 2 from China and 12 from USA), with six, six, and two of the isolates having both $s t x_{1 a}$ and $s t x_{2 a}$, $s t x_{2 c}$ and $s t x_{1 a}$, respectively (Table $S 8$ ). Studies reporting the recovery of STEC 0157:H7 from pigs are very limited. A number of studies on non-0157 STEC from pigs found $s x_{2 e}$ to be the predominant stx subtype ${ }^{9,19,75,76}$. The eae gene was present in all of the isolates included in this study except for Cat48, which is in agreement with the prevalence of this gene in STEC 0157 in published accounts ${ }^{73,77-83}$. All the isolates have the gene ehxA, located on the megaplasmid pO157 $7^{59}$. In addition, most of the STEC 0157:H7 isolates also harbor additional genes involved in intestinal adhesion (e.g. paa, toxB, and ehaA), encoding other toxins (e.g. ast $A$ ), involved in type II/III secretion system, and encoding fimbrial proteins. The distribution of these virulence genes does not seem to be associated with origin of isolation, i.e., pigs, cattle, or environment.

Only one (Pig04) of the 41 pig STEC 0157 isolates was predicted to be antibiotic-resistant. On the contrary, a relatively larger proportion of cattle isolates, $8 / 50$, harbored antibiotic resistance genes. Resistance to at least two antibiotic classes have been found for $16 \%$ of 0157 isolates from Alberta feedlots cattle ${ }^{84}$. Among non-type specific $E$. coli recovered from cattle in Alberta feedlots, the resistance to tetracyclines, aminoglycosides (represented by streptomycin), and sulfonamides (represented by 
sulfisoxazole) have been the most commonly detected ${ }^{85}$. The pattern of antibiotic resistance in environmental isolates was closely related to the phylogenetic group, with group 2,6, and 7 isolates and Env18 (from pig feces on farm 11) predicted to be resistant to at least one type of antibiotics. This suggests potential cross-contamination between two animal species as plant B processed both pork and beef and farm 10 had both cattle and swine during the period in which the isolates were recovered.

Unlike the plasmid p0157, other plasmids carried by $E$. coli 0157:H7 are not well studied. Rusconi et al. ${ }^{38}$ investigated the plasmids in STEC 0157:H7 associated with seven outbreaks in the USA and found plasmids of the incompatible groups Incl1, Incl2, IncK, IncFII, and pEC4115. The preliminary analysis in this study found the plasmids with replicon sequences Col (BS512), Col156, IncFII (pHN7A8), IncFII (pCoo), Incl2 (Delta), Incl1-I(Gamma), IncN and pEC4115 in STEC 0157:H7 isolates, with IncN, IncFII (pCoo) and IncFII (pHN7A8) plasmids carrying genes resistant to four, three and two/one classes of antibiotics including aminoglycosides, beta-lactams, sulfonamides, tetracyclines, and phenicols. Similar to the present pattern of antibiotic resistance genes, a larger proportion of cattle isolates carried additional plasmid(s).

In conclusion, pig and pork-production environmental STEC 0157:H7 isolates were classified mainly into seven distinct yet related phylogenetic groups, with each group primarily associated with the specific farm(s)/plant(s). The results demonstrated that some STEC 0157:H7 strains originating from pigs can establish persistence on farms, and that pigs can be a significant source for STEC 0157:H7 to contaminate pork, and of the environmental dissemination. Different antibiotic resistance and stx profiles were observed for STEC 0157:H7 isolates originating from Alberta cattle and pigs, with strains from the latter having a higher proportion of the more virulent $s x_{2 a}$ subtype.

\section{METHODS}

\section{Bacterial isolates}

A total of 121 STEC 0157:H7 isolates from pigs ( $n=41$; Pig01-41), porkproduction environments $(n=30$; Env01-30), and cattle $(n=50$; Cat01-50) in Alberta, Canada, were included in this study ${ }^{15,86-94}$ (Stanford et al., unpublished data; Reuter et al., unpublished data; Keenliside et al., unpublished data) (Table S1). Pig and cattle isolates were recovered from fecal samples of pigs and cattle, respectively. The environmental isolates were recovered from various types of samples collected from the pork production and distribution environments including retailers, pork processing facilities, and pig barns/lagoons. Among the environmental isolates, Env15 and Env26-Env30 (2014 outbreak), Env01 (2016), and Env02-Env05 (2018) were associated with the three E. coli 0157:H7 outbreaks attributed to contaminated pork in Alberta. Cattle isolates were recovered from the feces of cattle from various feedlots or at two slaughter plants in Alberta, Canada between 2002 and 2015. The pig and environmental isolates were from 11 farms (1-11) and four meat processing plants (A-D) between 2014 and 2018. We sequenced the genomes of Pig01-41, Cat01-12, Cat14-19, Cat21-23, Cat25-41, and Env01$25^{95}$. The genomes of Cat13, Cat20, Cat24, and Cat42-50 ${ }^{87,89}$ were downloaded from the National Center for Biotechnology Information (NCBI) GenBank database. For Env26-30 ${ }^{93}$, the raw sequencing data were downloaded from GenBank and assembled as described by Zhang, et al. ${ }^{95}$.

\section{Conventional genotyping using WGS data}

To determine the Manning clade of the isolates, the SNPs in four target genes including ECs2357, ECs2521, ECs3881, and ECs4130 were analyzed ${ }^{96}$. The clade for each isolate was then assigned as described previously ${ }^{96}$. For LSPA lineage, a perl script (https://github.com/egonozer/in_silico_pcr) and the primers designed by Yang et al. ${ }^{41}$. were used to perform in silico PCR. For MLST, mlst (https://github.com/tseemann/mlst) was used, with both Achtman's seven-gene scheme and Pasteur Institute's eight-gene scheme. The Clermont $E$. coli phylo-typing ${ }^{42}$, which assigns phylo-group to $E$. coli based on the presence/absence of the genes $\operatorname{arpA}$, chuA, and yjaA and an anonymous DNA fragment TspE4.C2, was also performed.

\section{Whole-genome SNP analysis and core SNP tree}

For SNP analysis, Snippy 4.4 .0 (https://github.com/tseemann/snippy) was used with the complete genome of EDL933 (ATCC 43895) as a reference (GenBank Accession no.: chromosome: CP008957.1, plasmid: CP008958.1) ${ }^{43}$. For all isolates except Cat13, Cat20, Cat24, and Cat42Cat50, trimmed sequencing reads were used as input for "snippy" with default settings. The isolates Cat13, Cat20, Cat24, and Cat42-Cat50 only had genome sequences available, and as such, -contigs options were used for "snippy". The contig-option shreds the contigs into synthetic reads and calls SNPs based on the generated reads. The recombination sites in the alignment file were removed using Gubbins ${ }^{97}$. Pairwise SNP distance matrix was generated using snp-dists (https://github.com/tseemann/snpdists). A best-scoring maximum likelihood tree based on core SNP alignment was constructed using RAxML version 8 with general time reversible gamma nucleotide model (GTRGAMMA) and 100 times of bootstrap analyses ${ }^{98}$. iTOL $v 4^{99}$ and the ggtree package ${ }^{100}$ in R were both used to display and annotate the phylogenetic tree.

\section{cgMLST and wgMLST}

ChewBBACA $^{101}$ was used to perform both CgMLST and wgMLST. The published Escherichia schemes were downloaded from Enterobase (http:// enterobase.warwick.ac.uk/schemes/). Both schemes were respectively adapted to chewBBACA and loci which did not meet the following criteria were removed: both valid start and stop codons were present in the coding sequence; the sequence length must be a multiple of 3 ; the sequence does not contain ambiguous character, i.e. containing ATGC only. The final schemes included 2513 and 24952 genes for cgMLST and wgMLST, respectively. The alleles were called with the default settings in chewBBACA. Neighbor joining trees were generated using GrapeTree ${ }^{102}$ based on the allele profiles of cgMLST and wgMLST, respectively, with the missing alleles ignored for pairwise comparison.

\section{Pan-genome analysis and gene content tree}

The 121 genomes were annotated using Prokka v1.13.7 ${ }^{103}$. The pangenome of the isolates was parsed using Roary ${ }^{104}$, with the identity threshold to cluster proteins set at $90 \%$. The functional category of the annotated coding sequences was assigned as described by Zhang et al. ${ }^{105}$. The Jaccard distances based on the presence or absence of genes between genomes were calculated using the dist() function in $\mathrm{R}$ with the method option set at "binary" ${ }^{106}$. A neighbor-joining tree was created using phangorn package ${ }^{107}$ in $\mathrm{R}$.

\section{Comparison of WGS-based subtyping methods}

To compare the four WGS subtyping methods, the distance matrices from four methods were used to generate UPGMA dendrograms via the hclust() function in R. For pairwise visual comparison, tanglegrams were created using the dendextend package in R. Cophenetic correlation coefficient was calculated for each pair of trees. To provide statistical support for the correlation, a permutation test was performed for each combination to produce one-sided $P$ values. If $P<0.05$, the similarity between two trees was regarded statistically significant.

\section{Virulence and antibiotic resistance genes}

The presence of $s t x$ and their subtypes were determined using the webserver of the Center for Genomic Epidemiology (CGE) (https://cge.cbs. dtu.dk/services/VirulenceFinder/) with $90 \%$ as the threshold for identity and $80 \%$ for the minimum coverage, respectively ${ }^{108}$. For other virulence genes, the reference database ecoli_vf (June 15, 2020; https://github.com/ phac-nml/ecoli_vf) and the virulence gene database (version, 2020-05-29) from CGE were both used. ABRicate v1.0.1 (https://github.com/tseemann/ abricate) was used to scan the contig sequences of each genome against the reference databases. A match with $>80 \%$ coverage and $>90 \%$ identity was regarded as positive. Antibiotic resistance genes and plasmid sequences were identified using ResFinder ${ }^{109}$ and PlasmidFinder ${ }^{58}$ in CGE, respectively.

\section{DATA AVAILABILITY}

The authors declare that the data supporting the findings of this study are presented within the manuscript and supplementary files. The raw sequencing data and 
genome sequences can be found at: https://www.ncbi.nlm.nih.gov/bioproject/ PRJNA661559.

Received: 25 November 2020; Accepted: 6 April 2021; Published online: 01 July 2021

\section{REFERENCES}

1. Gill, A. et al. Review of the state of knowledge on verotoxigenic Escherichia coli and the role of whole genome sequencing as an emerging technology supporting regulatory food safety in Canada. (2020).

2. Thorpe, C. M. Shiga toxin-producing Escherichia coli infection. Clin. Infect. Dis. 38, 1298-1303 (2004).

3. Valilis, E., Ramsey, A., Sidiq, S. \& DuPont, H. L. Non-O157 Shiga toxin-producing Escherichia coli-A poorly appreciated enteric pathogen: systematic review. Int. J. Infect. Dis. 76, 82-87 (2018)

4. Karmali, M. A., Steele, B. T., Petric, M. \& Lim, C. Sporadic cases of haemolyticuraemic syndrome associated with faecal cytotoxin and cytotoxin-producing Escherichia coli in stools. Lancet 1, 619-620 (1983).

5. O'Brien, A. O., Lively, T. A., Chen, M. E., Rothman, S. W. \& Formal, S. B. Escherichia coli 0157:H7 strains associated with haemorrhagic colitis in the United States produce a Shigella dysenteriae 1 (SHIGA) like cytotoxin. Lancet 1, 702 (1983).

6. Gill, A. \& Gill, C. O. Non-O157 verotoxigenic Escherichia coli and beef: a Canadian perspective. Can. J. Vet. Res 74, 161-169 (2010).

7. Heiman, K. E., Mody, R. K., Johnson, S. D., Griffin, P. M. \& Gould, L. H. Escherichia coli 0157 outbreaks in the United States, 2003-2012. Emerg. Infect. Dis. 21, 1293-1301 (2015).

8. Callaway, T. R., Carr, M. A., Edrington, T. S., Anderson, R. C. \& Nisbet, D. J. Diet, Escherichia coli O157:H7, and cattle: a review after 10 years. Curr. Issues Mol. Biol. 11, 67-79 (2009).

9. Tseng, M., Fratamico, P. M., Manning, S. D. \& Funk, J. A. Shiga toxin-producing Escherichia coli in swine: the public health perspective. Anim. Health Res. Rev. 15, 63-75 (2014).

10. Waddell, T. E., Coomber, B. L. \& Gyles, C. L. Localization of potential binding sites for the edema disease verotoxin (VT2e) in pigs. Can. J. Vet. Res. 62, 81-86 (1998).

11. Omer, M. K. et al. A systematic review of bacterial foodborne outbreaks related to red meat and meat products. Foodborne Pathog. Dis. 15, 598-611 (2018).

12. Honish, L. et al. Escherichia coli O157:H7 infections associated with contaminated pork products - Alberta, Canada, July-October 2014. Mmwr. Morbidity Mortal. Wkly. Rep. 65, 1477-1481 (2017).

13. AHS. E. coli outbreak linked to certain pork products in Alberta declared over, https://www.albertahealthservices.ca/news/releases/2018/Page14457.aspx (2018).

14. News, F. S. Alberta outbreak prompts raw pork and pork organ recall, https:// www.foodsafetynews.com/2016/02/alberta-e-coli-outbreak-prompts-raw-porkand-pork-organ-recall/ (2016).

15. Essendoubi, S. et al. Prevalence and characterization of Escherichia coli O157:H7 on pork carcasses and in swine colon content from provincially-licensed abattoirs in Alberta, Canada. J Food Prot, (2020).

16. Colello, R. et al. From farm to table: follow-up of Shiga toxin-producing Escherichia coli throughout the pork production chain in Argentina. Front Microbiol. 7, 93 (2016).

17. Tseng, M., Fratamico, P. M., Bagi, L., Manzinger, D. \& Funk, J. A. Shiga toxinproducing E. coli (STEC) in swine: prevalence over the finishing period and characteristics of the STEC isolates. Epidemiol. Infect. 143, 505-514 (2015).

18. Rajkhowa, S. \& Sarma, D. K. Prevalence and antimicrobial resistance of porcine O157 and non-0157 Shiga toxin-producing Escherichia coli from India. Trop. Anim. Health Prod. 46, 931-937 (2014).

19. Meng, Q. et al. Characterization of Shiga toxin-producing Escherichia coli isolated from healthy pigs in China. BMC Microbiol 14, 5 (2014).

20. Ho, W. S., Tan, L. K., Ooi, P. T., Yeo, C. C. \& Thong, K. L. Prevalence and characterization of verotoxigenic-Escherichia coli isolates from pigs in Malaysia. BMC Vet. Res. 9, 109 (2013).

21. Choi, Y. M. et al. Changes in microbial contamination levels of porcine carcasses and fresh pork in slaughterhouses, processing lines, retail outlets, and local markets by commercial distribution. Res. Vet. Sci. 94, 413-418 (2013).

22. Farzan, A., Friendship, R. M., Cook, A. \& Pollari, F. Occurrence of Salmonella, Campylobacter, Yersinia enterocolitica, Escherichia coli 0157 and Listeria monocytogenes in swine. Zoonoses Public Health 57, 388-396 (2010).

23. Lenahan, M. et al. The potential use of chilling to control the growth of Enterobacteriaceae on porcine carcasses and the incidence of $E$. coli O157:H7 in pigs. J. Appl. Microbiol. 106, 1512-1520 (2009).
24. Milnes, A. S. et al. Factors related to the carriage of Verocytotoxigenic E. coli, Salmonella, thermophilic Campylobacter and Yersinia enterocolitica in cattle, sheep and pigs at slaughter. Epidemiol. Infect. 137, 1135-1148 (2009).

25. Kaufmann, M. et al. Escherichia coli $\mathrm{O} 157$ and non-O157 Shiga toxin-producing Escherichia coli in fecal samples of finished pigs at slaughter in Switzerland. J. Food Prot. 69, 260-266 (2006).

26. Fratamico, P. M., Bagi, L. K., Bush, E. J. \& Solow, B. T. Prevalence and characterization of Shiga toxin-producing Escherichia coli in swine feces recovered in the National Animal Health Monitoring System's Swine 2000 study. Appl Environ. Microbiol 70, 7173-7178 (2004).

27. Bonardi, S. et al. Detection of Salmonella spp., Yersinia enterocolitica and verocytotoxin-producing Escherichia coli 0157 in pigs at slaughter in Italy. Int J. Food Microbiol 85, 101-110 (2003).

28. Eriksson, E., Nerbrink, E., Borch, E., Aspan, A. \& Gunnarsson, A. Verocytotoxinproducing Escherichia coli $0157: \mathrm{H} 7$ in the Swedish pig population. Vet. Rec. 152, 712-717 (2003).

29. Feder, I. et al. Isolation of Escherichia coli $0157: \mathrm{H} 7$ from intact colon fecal samples of swine. Emerg. Infect. Dis. 9, 380-383 (2003).

30. Johnsen, G., Wasteson, Y., Heir, E., Berget, O. I. \& Herikstad, H. Escherichia coli O157:H7 in faeces from cattle, sheep and pigs in the southwest part of Norway during 1998 and 1999. Int J. Food Microbiol 65, 193-200 (2001).

31. Leung, P. H., Yam, W. C., Ng, W. W. \& Peiris, J. S. The prevalence and characterization of verotoxin-producing Escherichia coli isolated from cattle and pigs in an abattoir in Hong Kong. Epidemiol. Infect. 126, 173-179 (2001).

32. Nakazawa, M. \& Akiba, M. Swine as a potential reservoir of Shiga toxinproducing Escherichia coli $0157: \mathrm{H7}$ in Japan. Emerg. Infect. Dis. 5, 833-834 (1999).

33. Chapman, P. A., Siddons, C. A., Gerdan Malo, A. T. \& Harkin, M. A. A 1-year study of Escherichia coli $\mathrm{O} 157$ in cattle, sheep, pigs and poultry. Epidemiol. Infect. 119, 245-250 (1997).

34. Tang, S. et al. Assessment and comparison of molecular subtyping and characterization methods for Salmonella. Front Microbiol. 10, 1591 (2019).

35. Schurch, A. C., Arredondo-Alonso, S., Willems, R. J. L. \& Goering, R. V. Whole genome sequencing options for bacterial strain typing and epidemiologic analysis based on single nucleotide polymorphism versus gene-by-gene-based approaches. Clin. Microbiol Infect. 24, 350-354 (2018).

36. McNally, A. et al. Combined analysis of variation in core, accessory and regulatory genome regions provides a super-resolution view into the evolution of bacterial populations. PLoS Genet. 12, e1006280 (2016).

37. Kaas, R. S., Friis, C., Ussery, D. W. \& Aarestrup, F. M. Estimating variation within the genes and inferring the phylogeny of 186 sequenced diverse Escherichia coli genomes. BMC Genomics 13, 577 (2012).

38. Rusconi, B. et al. Whole genome sequencing for genomics-guided investigations of Escherichia coli 0157:H7 outbreaks. Front Microbiol 7, 985 (2016).

39. Rumore, J. et al. Evaluation of whole-genome sequencing for outbreak detection of Verotoxigenic Escherichia coli 0157:H7 from the Canadian perspective. BMC Genomics 19, 870 (2018).

40. Manning, S. D. et al. Variation in virulence among clades of Escherichia coli O157: H7 associated with disease outbreaks. Proc. Natl Acad. Sci. USA 105, 4868-4873 (2008).

41. Yang, Z. et al. Identification of common subpopulations of non-sorbitol-fermenting, beta-glucuronidase-negative Escherichia coli 0157:H7 from bovine production environments and human clinical samples. Appl Environ. Microbiol. 70, 6846-6854 (2004).

42. Clermont, O., Christenson, J. K., Denamur, E. \& Gordon, D. M. The Clermont Escherichia coli phylo-typing method revisited: improvement of specificity and detection of new phylo-groups. Environ. Microbiol Rep. 5, 58-65 (2013).

43. Latif, H., Li, H. J., Charusanti, P., Palsson, B. O. \& Aziz, R. K. A gapless, unambiguous genome sequence of the enterohemorrhagic Escherichia coli 0157: H7 strain EDL933. Genome Announc. 2, e00821-14 (2014).

44. Sokal, R. R. \& Rohlf, F. J. The comparison of dendrograms by objective methods. Taxon 11, 33-40, (1962).

45. Pightling, A. W. et al. Interpreting whole-genome sequence analyses of foodborne bacteria for regulatory applications and outbreak investigations. Front Microbiol. 9, 1482 (2018).

46. Galperin, M. Y., Makarova, K. S., Wolf, Y. I. \& Koonin, E. V. Expanded microbial genome coverage and improved protein family annotation in the COG database. Nucleic acids Res. 43, D261-269 (2015).

47. Batisson, I. et al. Characterization of the novel factor paa involved in the early steps of the adhesion mechanism of attaching and effacing Escherichia coli. Infect. Immun. 71, 4516-4525 (2003).

48. Tatsuno, I. et al. toxB gene on pO157 of enterohemorrhagic Escherichia coli O157:H7 is required for full epithelial cell adherence phenotype. Infect. Immun. 69, 6660-6669 (2001). 
49. Wells, T. J. et al. EhaA is a novel autotransporter protein of enterohemorrhagic Escherichia coli $0157: \mathrm{H} 7$ that contributes to adhesion and biofilm formation. Environ. Microbiol. 10, 589-604 (2008).

50. Paton, A. W., Srimanote, P., Woodrow, M. C. \& Paton, J. C. Characterization of Saa, a novel autoagglutinating adhesin produced by locus of enterocyte effacementnegative Shiga-toxigenic Escherichia coli strains that are virulent for humans. Infect. Immun. 69, 6999-7009 (2001).

51. Galli, L., Torres, A. G. \& Rivas, M. Identification of the long polar fimbriae gene variants in the locus of enterocyte effacement-negative Shiga toxin-producing Escherichia coli strains isolated from humans and cattle in Argentina. FEMS Microbiol Lett. 308, 123-129 (2010).

52. Tarr, P. I. et al. Iha: a novel Escherichia coli 0157:H7 adherence-conferring molecule encoded on a recently acquired chromosomal island of conserved structure. Infect. Immun. 68, 1400-1407 (2000).

53. Stanley, P., Koronakis, V. \& Hughes, C. Acylation of Escherichia coli hemolysin: a unique protein lipidation mechanism underlying toxin function. Microbiol Mol. Biol. Rev. 62, 309-333 (1998).

54. Veilleux, S. \& Dubreuil, J. D. Presence of Escherichia coli carrying the EAST1 toxin gene in farm animals. Vet. Res 37, 3-13 (2006).

55. Savarino, S. J. et al. Enteroaggregative Escherichia coli heat-stable enterotoxin 1 represents another subfamily of E. coli heat-stable toxin. Proc. Natl Acad. Sci. USA 90, 3093-3097 (1993).

56. Paton, A. W., Srimanote, P., Talbot, U. M., Wang, H. \& Paton, J. C. A new family of potent $\mathrm{AB}(5)$ cytotoxins produced by Shiga toxigenic Escherichia coli. J. Exp. Med 200, 35-46 (2004)

57. Thomas, C. M. \& Summers, D. Encyclopedia of life sciences. (John Wiley \& Sons, Ltd, 2008).

58. Carattoli, A. et al. In silico detection and typing of plasmids using PlasmidFinder and plasmid multilocus sequence typing. Antimicrob. Agents Chemother. 58, 3895-3903 (2014).

59. Lim, J. Y., Yoon, J. \& Hovde, C. J. A brief overview of Escherichia coli O157:H7 and its plasmid 0157. J. Microbiol Biotechnol. 20, 5-14 (2010).

60. Kim, J. Y. et al. Isolation and identification of Escherichia coli O157:H7 using different detection methods and molecular determination by multiplex PCR and RAPD. J. Vet. Sci. 6, 7-19 (2005).

61. Jaros, P. et al. Geographic divergence of bovine and human Shiga toxin-producing Escherichia coli O157: H7 genotypes. NZ 20, 1980 (2014).

62. Mellor, G. E. et al. Geographically distinct Escherichia coli $\mathrm{O} 157$ isolates differ by lineage, Shiga toxin genotype, and total shiga toxin production. J. Clin. Micro. 53, 579-586 (2015).

63. Pianciola, L. \& Rivas, M. Genotypic features of clinical and bovine Escherichia coli 0157 strains isolated in countries with different associated-disease incidences. Microorganisms 6, 36 (2018)

64. Strachan, N. J. et al. Whole genome sequencing demonstrates that geographic variation of Escherichia coli 0157 genotypes dominates host association. Sci. Rep. 5, 14145 (2015).

65. Touchon, M. et al. Organised genome dynamics in the Escherichia coli species results in highly diverse adaptive paths. PLoS Genet. 5, e1000344 (2009).

66. Wochtl, B. et al. Comparison of clinical and immunological findings in gnotobiotic piglets infected with Escherichia coli O104:H4 outbreak strain and EHEC O157:H7. Gut Pathog. 9, 30 (2017).

67. Booher, S. L., Cornick, N. A. \& Moon, H. W. Persistence of Escherichia coli O157:H7 in experimentally infected swine. Vet. Microbiol. 89, 69-81 (2002).

68. Moxley, R. A. Edema disease. Vet. Clin. North Am. Food Anim. Pr. 16, 175-185 (2000)

69. Melton-Celsa, A. R. Shiga toxin (Stx) classification, structure, and function. Microbiol Spectr. 2, EHEC-0024-2013 (2014).

70. Fuller, C. A., Pellino, C. A., Flagler, M. J., Strasser, J. E. \& Weiss, A. A. Shiga toxin subtypes display dramatic differences in potency. Infect. Immun. 79, 1329-1337 (2011).

71. Tesh, V. L. et al. Comparison of the relative toxicities of Shiga-like toxins type I and type II for mice. Infect. Immun. 61, 3392-3402 (1993).

72. Tarr, G. A. M. et al. Contribution and interaction of Shiga toxin genes to Escherichia coli 0157:H7 virulence. Toxins (Basel) 11, 607 (2019).

73. Chui, L. et al. Molecular profiling of Escherichia coli O157:H7 and non-O157 strains isolated from humans and cattle in Alberta, Canada. J. Clin. Microbiol. 53, 986-990 (2015)

74. Goma, M. K. E., Indraswari, A., Haryanto, A. \& Widiasih, D. A. Detection of Escherichia coli O157:H7 and Shiga toxin 2a gene in pork, pig feces, and clean water at Jagalan slaughterhouse in Surakarta, Central Java Province, Indonesia. Vet. World 12, 1584-1590 (2019).

75. Baranzoni, G. M. et al. Characterization of Shiga toxin subtypes and virulence genes in porcine Shiga toxin-producing Escherichia coli. Front Microbiol. 7, 574 (2016).
76. Mohlatlole, R. P. et al. Virulence profiles of enterotoxigenic, Shiga toxin and enteroaggregative Escherichia coli in South African pigs. Trop. Anim. Health Prod. 45, 1399-1405 (2013).

77. Blanco, M. et al. Serotypes, virulence genes, and intimin types of Shiga toxin (verotoxin)-producing Escherichia coli isolates from cattle in Spain and identification of a new intimin variant gene (eae-xi). J. Clin. Microbiol. 42, 645-651 (2004).

78. Kobayashi, N. et al. Virulence gene profiles and population genetic analysis for exploration of pathogenic serogroups of Shiga toxin-producing Escherichia coli. J. Clin. Microbiol. 51, 4022-4028 (2013).

79. Meng, J., Zhao, S. \& Doyle, M. P. Virulence genes of Shiga toxin-producing Escherichia coli isolated from food, animals and humans. Int J. Food Microbiol $\mathbf{4 5}$, 229-235 (1998).

80. Mora, A. et al. Phage types, virulence genes and PFGE profiles of Shiga toxinproducing Escherichia coli 0157:H7 isolated from raw beef, soft cheese and vegetables in Lima (Peru). Int J. Food Microbiol. 114, 204-210 (2007).

81. Sallam, K. I., Mohammed, M. A., Ahdy, A. M. \& Tamura, T. Prevalence, genetic characterization and virulence genes of sorbitol-fermenting Escherichia coli O157:H- and E. coli O157:H7 isolated from retail beef. Int J. Food Microbiol 165 295-301 (2013).

82. Solomakos, N. et al. Occurrence, virulence genes and antibiotic resistance of Escherichia coli 0157 isolated from raw bovine, caprine and ovine milk in Greece. Food Microbiol. 26, 865-871 (2009)

83. Tóth, I. et al. Virulence genes and molecular typing of different groups of Escherichia coli 0157 strains in cattle. Appl. Environ. Microbiol. 75, 6282 (2009).

84. Rao, S. et al. Antimicrobial drug use and antimicrobial resistance in enteric bacteria among cattle from Alberta feedlots. Foodborne Pathog. Dis. 7, 449-457 (2010).

85. Benedict, K. M. et al. Antimicrobial resistance in Escherichia coli recovered from feedlot fattle and associations with antimicrobial use. PLOS ONE 10, e0143995 (2015).

86. Stanford, K., Johnson, R. P., Alexander, T. W., McAllister, T. A. \& Reuter, T. Influence of season and feedlot location on prevalence and virulence factors of seven serogroups of Escherichia coli in feces of western-Canadian slaughter cattle. PLOS ONE 11, e0159866 (2016).

87. Mercer, R. G. et al. Genetic determinants of heat resistance in Escherichia coli. Front Microbiol. 6, 932 (2015).

88. Stanford, K. et al. Monitoring Escherichia coli O157:H7 in inoculated and naturally colonized feedlot cattle and their environment. J. Food Prot. 68, 26-33 (2005)

89. Munns, K. D. et al. Comparative genomic analysis of Escherichia coli 0157:H7 isolated from super-shedder and low-shedder cattle. PLOS ONE 11, e0151673 (2016).

90. Bach, S. J. et al. Electrolyzed oxidizing anode water as a sanitizer for use in abattoirs. J. Food Prot. 69, 1616-1622 (2006).

91. Stanford, K., Gibb, D. \& McAllister, T. A. Evaluation of a shelf-stable direct-fed microbial for control of Escherichia coli 0157 in commercial feedlot cattle. Can. J. Anim. Sci. 93, 535-542 (2013).

92. Stanford, K., Hannon, S., Booker, C. W. \& Jim, G. K. Variable efficacy of a vaccine and direct-fed microbial for controlling Escherichia coli O157:H7 in feces and on hides of feedlot cattle. Foodborne Pathog. Dis. 11, 379-387 (2014).

93. Berenger, B. M. et al. The utility of multiple molecular methods including whole genome sequencing as tools to differentiate Escherichia coli 0157:H7 outbreaks. Euro Surveill. 20, 30073 (2015).

94. Stephens, T. P., McAllister, T. A. \& Stanford, K. Perineal swabs reveal effect of super shedders on the transmission of Escherichia coli 0157:H7 in commercial feedlots. J. Anim. Sci. 87, 4151-4160 (2009).

95. Zhang, P. et al. Genome sequences of 104 Escherichia coli O157:H7 isolates from pigs, cattle, and pork production environments in Alberta, Canada. Microbiol. Resour. Announc. 10, (2021).

96. Riordan, J. T., Viswanath, S. B., Manning, S. D. \& Whittam, T. S. Genetic differentiation of Escherichia coli $\mathrm{O} 157: \mathrm{H} 7$ clades associated with human disease by real-time PCR. J. Clin. Microbiol. 46, 2070-2073 (2008)

97. Croucher, N. J. et al. Rapid phylogenetic analysis of large samples of recombinant bacterial whole genome sequences using Gubbins. Nucleic Acids Res. 43, e15-e15 (2014).

98. Stamatakis, A. RAxML version 8: a tool for phylogenetic analysis and postanalysis of large phylogenies. Bioinforma. (Oxf., Engl.) 30, 1312-1313 (2014).

99. Letunic, I. \& Bork, P. Interactive Tree Of Life (iTOL) v4: recent updates and new developments. Nucleic acids Res. 47, W256-W259 (2019).

100. Yu, G. Using ggtree to visualize data on tree-like structures. Curr. Protoc. Bioinform. 69, e96 (2020).

101. Silva, M. et al. chewBBACA: A complete suite for gene-by-gene schema creation and strain identification. Micro. Genom. 4, e000166 (2018)

102. Zhou, Z. et al. GrapeTree: visualization of core genomic relationships among 100,000 bacterial pathogens. Genome Res. 28, 1395-1404 (2018). 
103. Seemann, T. Prokka: rapid prokaryotic genome annotation. Bioinformatics (Oxf., Engl.) 30, 2068-2069 (2014).

104. Page, A. J. et al. Roary: rapid large-scale prokaryote pan genome analysis. Bioinformatics (Oxf., Engl.) 31, 3691-3693 (2015).

105. Zhang, P., Gänzle, M. \& Yang, X. Complementary antibacterial effects of bacteriocins and organic acids as revealed by comparative analysis of Carnobacterium spp. from meat. Appl. Environ. Microbiol. 85, e01227-19 (2019).

106. Zheng, J., Zhao, X., Lin, X. B. \& Ganzle, M. Comparative genomics Lactobacillus reuteri from sourdough reveals adaptation of an intestinal symbiont to food fermentations. Sci. Rep. 5, 18234 (2015).

107. Schliep, K., Potts, A. J., Morrison, D. A. \& Grimm, G. W. Intertwining phylogenetic trees and networks. Methods Ecol. Evol. 8, 1212-1220 (2017).

108. Joensen, K. G. et al. Real-time whole-genome sequencing for routine typing, surveillance, and outbreak detection of verotoxigenic Escherichia coli. J. Clin. Microbiol. 52, 1501-1510 (2014)

109. Bortolaia, V. et al. ResFinder 4.0 for predictions of phenotypes from genotypes. J. Antimicrob. Chemother. 75, 3491-3500 (2020).

\section{ACKNOWLEDGEMENTS}

Alberta Agriculture and Forestry and Alberta Innovates are acknowledged for funding (2019F108R). Dr. Lisa Tymensen and Dr. Gary Gensler are acknowledged for their assistance on project planning and bacteria shipping. The bioinformatics analysis was performed using the high performance computing servers hosted by Agriculture and Agri-Food Canada through the Biocluster and Compute Canada.

\section{AUTHOR CONTRIBUTIONS}

PZ: formal analysis, methodology and writing the original draft; SE: conceptualization, funding acquisition, and review and editing manuscript; JK: funding acquisition, and review and editing manuscript; TR: funding acquisition, methodology, and review and editing manuscript; KS: funding acquisition, methodology, and review and editing manuscript; RK: methodology and review and editing manuscript;
PL: methodology and review and editing manuscript; $X Y$ : conceptualization, funding acquisition, supervision, project administration, and review and editing manuscript.

\section{COMPETING INTERESTS}

The authors declare no competing interests.

\section{ADDITIONAL INFORMATION}

Supplementary information The online version contains supplementary material available at https://doi.org/10.1038/s41538-021-00097-0.

Correspondence and requests for materials should be addressed to X.Y.

Reprints and permission information is available at http://www.nature.com/ reprints

Publisher's note Springer Nature remains neutral with regard to jurisdictional claims in published maps and institutional affiliations.

(c) (i) Open Access This article is licensed under a Creative Commons Attribution 4.0 International License, which permits use, sharing, adaptation, distribution and reproduction in any medium or format, as long as you give appropriate credit to the original author(s) and the source, provide a link to the Creative Commons license, and indicate if changes were made. The images or other third party material in this article are included in the article's Creative Commons license, unless indicated otherwise in a credit line to the material. If material is not included in the article's Creative Commons license and your intended use is not permitted by statutory regulation or exceeds the permitted use, you will need to obtain permission directly from the copyright holder. To view a copy of this license, visit http://creativecommons.org/licenses/by/4.0/.

(c) Crown 2021, corrected publication 2021 